\title{
TOWARD MOTIVIC INTEGRATION OVER WILD DELIGNE-MUMFORD STACKS
}

\author{
TAKEHIKO YASUDA
}

To Professor Yujiro Kawamata

\begin{abstract}
We discuss how the motivic integration will be generalized to wild Deligne-Mumford stacks, that is, stabilizers may have order divisible by the characteristic of the base or residue field. We pose several conjectures on this topic. We also present some possible applications concerning stringy invariants, resolution of singularities, and weighted counts of extensions of local fields.
\end{abstract}

\section{Contents}

1. Introduction

2. The ring of values

3. Motivic integration over varieties

4. Motivic integration over Deligne-Mumford stacks

5. Formulating the change of variables formula for DM stacks

6. More details on the linear case

7. Untwisting: a justification of conjectures

8. Stringy motifs vs. motivic masses of $G$-covers

9. Equisingular families and uniformity of motivic masses 18

10. Non-existence of resolution of singularities 20

References

\section{INTRODUCTION}

The aim of this paper is to present an attempt to generalize the motivic integration to wild Deligne-Mumford stacks. In relation to the McKay correspondence, Denef and Loeser 9 ] developed the motivic integration applicable to the quotient map $\mathbb{A}_{k}^{d} \rightarrow \mathbb{A}_{k}^{d} / G$ for a finite group $G \subset G L_{d}(k)$ with $k$ a field of characteristic zero. Motivated by this, the author [31, 30] developed the motivic integration over Deligne-Mumford stacks under some tameness condition. In this paper, we will make conjectures on how the theory will be generalized by dropping the tameness condition. The easiest wild case where $G$ has order equal to the characteristic has been already studied in 32 .

Let $A$ be a complete discrete valuation ring with algebraically closed residue field and $D:=\operatorname{Spec} A$. For a Deligne-Mumford stack $\mathcal{X}$ of finite type over $D$, we will define a twisted arc of $\mathcal{X}$ as a representable $D$-morphism $\mathcal{E} \rightarrow \mathcal{X}$, where $\mathcal{E}$ is the

This work was supported by Grants-in-Aid for Scientific Research (22740020). 
quotient stack associated to some Galois cover of $D$. We expect that we can develop the motivic integration on the space of twisted $\operatorname{arcs}$ of $\mathcal{X}$, which we will denote by $\mathcal{J}_{\infty} \mathcal{X}$. Our main conjecture is the following:

Conjecture 1.1 (Conjecture 5.5). For a proper birational morphism $f: \mathcal{Y} \rightarrow \mathcal{X}$ of pure-dimensional Deligne-Mumford stacks of finite type over D, we have a natural map $f_{\infty}: \mathcal{J}_{\infty} \mathcal{Y} \rightarrow \mathcal{J}_{\infty} \mathcal{X}$. Then, for a measurable function $F$ on a subset $C \subset \mathcal{J}_{\infty} \mathcal{X}$, we have the change of variables formula

$$
\int_{C} \mathbb{L}^{F+w_{\mathcal{X}}} d \mu_{\mathcal{X}}=\int_{f_{\infty}^{-1}(C)} \mathbb{L}^{F \circ f_{\infty}-\mathrm{ord} \mathrm{Jac}_{f}+w_{\mathcal{Y}}} d \mu_{\mathcal{Y}} .
$$

Here ord $\operatorname{Jac}_{f}$ is the Jacobian order function of $f$ and $w_{\mathcal{X}}$ and $w_{\mathcal{Y}}$ are canonically defined weight functions on $\mathcal{J}_{\infty} \mathcal{X}$ and $\mathcal{J}_{\infty} \mathcal{Y}$ respectively.

In this paper, we will try to justify the conjecture. To do so, our main tool is what we call untwisting, a technique reducing twisted arcs to non-twisted ones. We will study the formula in more detail when $\mathcal{Y}$ is the quotient stack $\left[\mathbb{A}_{D}^{d} / G\right]$ and $\mathcal{X}$ is the quotient variety $\mathbb{A}_{D}^{d} / G$ for some linear action $G \curvearrowright \mathbb{A}_{D}^{d}$ of a finite group $G$. In this case, we will make a more explicit and conjectural expression of the weight function.

As an application, we will reach another conjecture which ties stringy invariants of quotient singularities with mass formulae for extensions of local fields. For a local field $K$ with residue field having $q$ elements, Serre 23 . proved a mass formula

$$
\sum_{L} \frac{1}{\sharp \operatorname{Aut}(L / K)} \cdot q^{-d(L)}=q^{1-n},
$$

where $L$ runs over isomorphism classes of totally ramified field extensions $L / K$ of degree $n$ and $d(L)$ the discriminant exponent of $L / K$. Bhargava [5] proved an analogous mass formula for all étale extensions of fixed degree. Also we may consider the motivic version of such counts, replacing $q$ with $\mathbb{L}$ and summation with motivic integration over the space of extensions. From our conjectural change of variables formula, we will derive an equality between a stringy invariant of the associated quotient singularity and some motivic count of extensions of the local field, the fraction field of $A$. We may regard this as a version of the McKay correspondence. Kedlaya [13] and then Wood [25] worked on this kind of counting problems in terms of local Galois representations. Their works might have a more direct relation with our motivic count 1

Another possible application concerns resolution of singularities. Stringy invariants have a lot of information on resolution of singularities. Our change of variables formula would be helpful in reducing stringy invariants for varieties with quotient singularities to those for smooth Deligne-Mumford stacks, which might be easier to compute. An existence of resolution impose some constraints on stringy invariants. Thus, if we find a singular variety with stringy invariant violating one of them, then we can prove the non-existence of resolution of singularities? Similar arguments can apply to the problem on the non-existence of crepant resolution.

We will also discuss the problem when a family of quotient singularities has a constant stringy invariant, especially in the case where the family contains both tame and wild ones.

\footnotetext{
${ }^{1}$ Afterwards such a relation was studied in [27, 26].

${ }^{2}$ Afterwards this viewpoint was adopted in [26].
} 
There have been considerable developments [27, 26, 28, 29] on the subject after the acceptance of the paper for publication until the final proof. I add several footnotes to mention them. All footnotes in the paper are added at the final proof.

1.1. Acknowledgements. The author wish to thank Tomoyoshi Ibukiyama and Seidai Yasuda for letting me know the relevance of Serre's result [23] in this work. The author also like to thank Shuji Saito for stimulating discussion on quotient singularities, Kiran S. Kedlaya, Julien Sebag and Melanie Matchett Wood for reading a draft of the paper and giving me useful comments, and Fabio Tonini for kindly explaining his result on the moduli of ramified covers.

1.2. Conventions. We fix an algebraically closed field $k$, a complete discrete valuation ring $A$ with residue field $k$. We denote the fraction field of $A$ by $K$. We put $D:=\operatorname{Spec} A$ and $D^{*}:=\operatorname{Spec} K$ : we call them a formal disk and punctured formal disk respectively. Always $G$ will denote a finite group.

As many of our statements will be conjectural, we will often make heuristic arguments rather than rigorous ones. For this reason, we often identify a $k$-scheme with its $k$-point set.

\section{The RING OF VALUes}

Motivic measures and integrals usually take values in some extension of the Grothendieck ring of varieties. There are several versions of such extensions which are slightly different one another. Throughout the paper, we fix an appropriate one and denote it by $\hat{\mathcal{M}}=\hat{\mathcal{M}}_{k}$. The following are some of its properties which will be necessary in subsequent sections:

(1) $\hat{\mathcal{M}}$ has an element associated to each scheme $X$ of finite type over $k$, denoted $[X]$. We write $\mathbb{L}:=\left[\mathbb{A}_{k}^{1}\right]$.

(2) $\hat{\mathcal{M}}$ contains the fractional powers $\mathbb{L}^{a}, a \in \mathbb{Q}$ of $\mathbb{L}$.

(3) For a bijective morphism $Y \rightarrow X,[Y]=[X]$. In particular, $[X]=\left[X_{\text {red }}\right]$. Also, if $Y \subset X$ is a closed subset, then $[X]=[Y]+[X \backslash Y]$. This enables us to determine $[C] \in \hat{\mathcal{M}}$ for a constructible subset $C$ of a variety.

(4) Let $f: Y \rightarrow X$ be a morphism such that every fiber $f^{-1}(x)$ admits a bijective morphism from or to the quotient variety $\mathbb{A}_{k}^{n} / H$ for some fixed $n$ and for some linear action $H \curvearrowright \mathbb{A}_{k}^{n}$ of a finite group $H$. (We will call $f$ an $\mathbb{L}^{n}$-fibration.) Then $[Y]=[X] \mathbb{L}^{n}$.

(5) For $r \in \mathbb{Z}_{>0}$ and a countable index set $I$, an infinite sum of the form

$$
\sum_{i \in I}\left[X_{i}\right] \mathbb{L}^{a_{i}}, a_{i} \in \frac{1}{r} \mathbb{Z}
$$

converges if and only if for every $a \in \mathbb{R}$, there exists at most finitely many $i$ such that $\operatorname{dim} X_{i}+a_{i} \geq a$.

(6) There exists a ring homomorphism

$$
P: \hat{\mathcal{M}} \rightarrow \bigcup_{r=1}^{\infty} \mathbb{Z}\left(\left(T^{-1 / r}\right)\right)
$$

called the virtual Poincaré realization. For a variety $X, P([X])$ equals the virtual Poincaré polynomial $P(X)$ of $X$. If $X$ is smooth and proper, then $P(X)=\sum_{i}(-1)^{i} b_{i}(X) T^{i}$, where $b_{i}(X)$ is the $i$-th Betti number for $l$-adic cohomology. Also we have $P(\mathbb{L})=T^{2}$. 
Concerning the construction of $\hat{\mathcal{M}}$, Properties (11) and (2) determine generators, (31) and (4) relations and (5) how to complete the ring. Property (6) is rather a consequence of the construction. For instance, see 32] for details.

\section{Motivic integration OVER VARIEties}

In this section, we will briefly recall the motivic integration invented by Kontsevich, developed by Denef and Loeser [8, 9] in characteristic zero, and Sebag [22] (see also [18]) in positive or mixed characteristic.

3.1. Varieties over $k$. Let $X$ be a variety of pure dimension $d$ over $k$. For a nonnegative integer $n$, the $n$-jet scheme of $X$, denoted $J_{n} X$, is the fine moduli scheme parameterizing $n$-jets:

$$
J_{n} X=\left\{\operatorname{Spec} k[t] /\left(t^{n+1}\right) \rightarrow X\right\} .
$$

They are schemes of finite type over $k$. For $n \geq m$, we have a natural morphism $J_{n} X \rightarrow J_{m} X$, called the truncation. The arc space of $X$, denoted $J_{\infty} X$, is the projective limit of $J_{n} X, n \in \mathbb{Z}_{\geq 0}$, which parameterizes arcs:

$$
J_{\infty} X=\{\operatorname{Spec} k[[t]] \rightarrow X\} .
$$

The natural maps $\pi_{n}: J_{\infty} X \rightarrow J_{n} X$ are also called truncations.

The arc space has a measure taking values in $\hat{\mathcal{M}}$, which is called the motivic measure. We denote it by $\mu_{X}$ and define it as follows: a subset $C \subset J_{\infty} X$ is said to be stable if for some $n$,

(1) $\pi_{n}(C)$ is a constructible subset,

(2) $C=\pi_{n}^{-1}\left(\pi_{n}(C)\right)$, and

(3) for every $n^{\prime} \geq n, \pi_{n^{\prime}+1}(C) \rightarrow \pi_{n}(C)$ is an $\mathbb{L}^{d}$-fibration.

For a stable subset $C$, we put

$$
\mu_{X}(C):=\left[\pi_{n}(C)\right] \mathbb{L}^{-n d} \in \hat{\mathcal{M}},(n \gg 0) .
$$

This defines the motivic measure on stable subsets. We can extend this to a larger class of subsets called measurable subsets. Roughly, a measurable subset is a subset of $J_{\infty} X$ which can be approximated by a series of stable subsets. Let

$$
F: J_{\infty} X \supset C \rightarrow \frac{1}{r} \mathbb{Z} \cup\{\infty\}
$$

be a measurable function on a subset $C$ for some $r \in \mathbb{Z}_{>0}$, that is, every fiber of it is a measurable subset and $F^{-1}(\infty)$ has measure zero. Then we define the motivic integral of $\mathbb{L}^{F}$ by

$$
\int_{C} \mathbb{L}^{F} d \mu_{X}:=\sum_{n \in \frac{1}{r} \mathbb{Z}} \mu_{X}\left(F^{-1}(n)\right) \mathbb{L}^{n} \in \hat{\mathcal{M}} \cup\{\infty\} .
$$

Here, if the infinite sum diverges, then we put the integral to be $\infty$.

3.2. Varieties over $D$. All these about the motivic integration over $k$-varieties can be generalized to $D$-varieties. Let $X$ be a $D$-variety, that is, an integral scheme of finite type over $D$. For $n \in \mathbb{Z}_{\geq 0}$, we put $A_{n}:=A / \mathfrak{m}^{n+1}, D_{n}:=\operatorname{Spec} A_{n}$. In this setting, we put

$$
J_{n} X=\left\{D \text {-morphisms } D_{n} \rightarrow X\right\},
$$


which is a scheme of finite type over $k$ (not $D$ ). (The functor represented by this scheme is called a Greenberg functor.) Then $J_{\infty} X$ is again the projective limit of $J_{n} X, n \geq 0$ and

$$
J_{\infty} X=\{D \text {-morphisms } D \rightarrow X\} .
$$

Let us suppose also that $X$ is flat and of relative dimension $d$ over $D$. Then, in the same way as in the case of $k$-varieties, we can define the motivic measure on $J_{\infty} X$ and the motivic integral $\int_{C} \mathbb{L}^{F} d \mu_{X}$ for a measurable function $F$ on a subset $C$ of $J_{\infty} X$. The motivic integration for a $k$-variety $X$ is equivalent to the one for the induced $D$-variety $X \times_{k} D$ with $D=\operatorname{Spec} k[[t]]$.

\section{Motivic integration over Deligne-Mumford stacks}

4.1. Spaces of $G$-covers of a formal disk. We mean by a $G$-cover of $D^{*}$ an étale $G$-torsor $E^{*} \rightarrow D^{*}$. Then a $G$-cover of $D$ is the finite cover $E \rightarrow D$ associated to a $G$-cover $E^{*} \rightarrow D^{*}$ of $D^{*}$. Here $E$ is the unique normal scheme finite over $D$ containing $E^{*}$ as an open dense subscheme. Let $\bar{K}$ be the algebraic closure of $K$. A $G$-cover $E^{(*)} \rightarrow D^{(*)}$ is called pointed if a lift of the natural $\bar{K}$-point of $D^{(*)}$ to $E^{(*)}$ is prescribed.

Conjecture 4.1. There exist the moduli spaces parameterizing isomorphism classes of the pointed/unpointed $G$-covers of $D$ : we will denote them by $G-\mathrm{Cov}^{\mathrm{pt}}(D)$ and $G-\operatorname{Cov}(D)$ respectively.

The group $G$ acts on $G$-Cov ${ }^{\mathrm{pt}}(D)$ by changing the given pointing or equivalently by replacing the given $G$-action on $E$ with conjugation. Then we should have

$$
G-\operatorname{Cov}^{\mathrm{pt}}(D) / G=G-\operatorname{Cov}(D) .
$$

The conjecture holds if $\operatorname{char}(k) \nmid \sharp G$. Indeed the moduli spaces are just finite points in this case. Moreover $G-\operatorname{Cov}(D) \cong G$ - $\operatorname{Cov}\left(D^{*}\right)$ has exactly $\sharp \operatorname{Conj}(G)$ points. When $A$ has equal characteristic $p>0$ and $G$ is a $p$-group, Harbater [10] constructed the (coarse) moduli spaces of $G$-covers of $D^{*}$. If $G_{K}$ denotes the absolute Galois group of $K$, then there exists a one-to-one correspondence between $G$-Cov ${ }^{\mathrm{pt}}(D)$ and the set of continuous homomorphisms $G_{K} \rightarrow G$. Actually the conjecture above seems to be one of the technical keys of the whole story in this paper. For instance, the existence of moduli spaces of $G$-jets/arcs and twisted jets/arcs discussed below would easily follow from the conjecture along the same line as in 32. A recent result by Tonini 24 may be helpful in addressing this problem.

Besides Conjecture 4.1, we also expect that $G-\operatorname{Cov}(D)$ is the inductive limit of some series

$$
V_{0} \rightarrow V_{1} \rightarrow V_{2} \rightarrow \cdots
$$

such that $V_{i}$ are schemes of finite type and $V_{i} \rightarrow V_{i+1}$ are injective morphisms. (We expect them to be not immersions but rather something like immersions followed by Frobenius morphisms, as such a phenomenon appears in 10.) We will say that a subset of $G-\operatorname{Cov}(D)$ is constructible if it is the image of a constructible subset of some $V_{i}$. To a constructible subset $C$ of $G-\operatorname{Cov}(D)$, we associate $[C] \in \hat{\mathcal{M}}$ in the obvious way. We obtain the tautological motivic measure on $G$-Cov $(D)$ :

$$
\begin{aligned}
\tau:\{\text { constructible subsets of } G-\operatorname{Cov}(D)\} & \rightarrow \hat{\mathcal{M}} \\
C & \mapsto \tau(C)=[C]
\end{aligned}
$$


4.2. $G$-arcs and jets. Let $M$ be a $D$-variety endowed with a $G$-action.

Definition 4.2. We define a $G$-arc of $M$ as a $G$-equivariant $D$-morphism $E \rightarrow M$ for some $G$-cover $E \rightarrow D$. Two $G$-arcs $E, E^{\prime} \rightarrow M$ are isomorphic if there exists an isomorphism $E \rightarrow E^{\prime}$ of $G$-covers compatible with the given morphisms $E, E^{\prime} \rightarrow M$.

For a connected Galois cover $E \rightarrow D$ of degree $e$, we define a closed subscheme $E_{n}$ of $E$ to be the one having length $1+n e$. In particular, $E_{0} \cong \operatorname{Spec} k$. For a non-connected Galois cover $E=\bigsqcup_{i} E^{i} \rightarrow D$ with $E^{i}$ connected components, we put $E_{n}:=\bigsqcup_{i} E_{n}^{i}$.

Definition 4.3. We define a $G$-n-jet of $M$ as a pair $\left(E, E_{n} \rightarrow M\right)$ of a $G$-cover $E \rightarrow D$ and a $G$-equivariant $D$-morphism $E_{n} \rightarrow M$. Two $G$ - $n$-jets $\left(E, E_{n} \rightarrow M\right)$ and $\left(E^{\prime}, E_{n}^{\prime} \rightarrow M\right)$ are isomorphic if there exists an isomorphism $E \rightarrow E^{\prime}$ of $G$ covers such that the induced isomorphism $E_{n} \rightarrow E_{n}^{\prime}$ is compatible with the given morphisms to $M$.

Conjecture 4.4. There exist moduli spaces parameterizing isomorphism classes of $G$-arcs and $G$-n-jets of $M$ for every n: we will denote them by $J_{\infty}^{G} M$ and $J_{n}^{G} M$ respectively.

If this is the case, we would have natural morphisms

$$
J_{\infty}^{G} M \rightarrow \cdots \rightarrow J_{n+1}^{G} M \rightarrow J_{n}^{G} M \rightarrow \cdots \rightarrow J_{0}^{G} M \rightarrow G-\operatorname{Cov}(D) .
$$

For $E \in G$ - $\operatorname{Cov}(D)$ and for each $n$, we put $J_{n}^{G, E} M \subset J_{n}^{G} M$ to be the fiber over $E$.

Lemma 4.5. Let $E \in G-\operatorname{Cov}(D)$ and $G^{\prime} \subset G$ the stabilizer of a connected component $E^{\prime}$ of $E$, that is, $G^{\prime}:=\left\{g \in G \mid g\left(E^{\prime}\right)=E^{\prime}\right\}$. Let $M_{0}:=M \times_{D} D_{0}$. (Recall $D_{0}=$ Spec $k$.) Then

$$
J_{0}^{G, E} M \cong M_{0}^{G^{\prime}} / N_{G}\left(G^{\prime}\right)
$$

Here $N_{G}\left(G^{\prime}\right)$ is the normalizer of $G^{\prime}$ in $G$.

Proof. A pointed $G$-0-jet $\alpha: E_{0} \rightarrow M_{0}$ is uniquely determined by $\alpha\left(E_{0}^{\prime}\right) \in M_{0}$, which is invariant under the $G^{\prime}$-action. Changing the connected component, $\alpha\left(E_{0}^{\prime}\right)$ will be changed to a point of $M_{0}$ invariant under a subgroup conjugate to $G^{\prime}$. Thus we have

$$
J_{0}^{G, E} M \cong\left(\bigcup_{H: \text { conjugate to } G^{\prime}} M_{0}^{H}\right) / G \cong M_{0}^{G^{\prime}} / N_{G}\left(G^{\prime}\right) .
$$

4.3. Twisted arcs and jets. Let $\mathcal{X}$ be a DM stack of finite type over $D$.

Definition 4.6. A stacky formal disk is a connected and normal DM stack $\mathcal{E}$ which is birational and finite over $D$. A twisted arc of $\mathcal{X}$ is a representable $D$-morphism $\mathcal{E} \rightarrow \mathcal{X}$ with $\mathcal{E}$ a stacky formal disk. (Note that $\mathcal{E} \rightarrow \mathcal{X}$ is representable if and only if the induced map of stabilizers of closed points is injective.) Two twisted arcs $\mathcal{E} \rightarrow \mathcal{X}$ and $\mathcal{E}^{\prime} \rightarrow \mathcal{X}$ are isomorphic if there exists an isomorphism $\mathcal{E} \rightarrow \mathcal{E}^{\prime}$ making the diagram

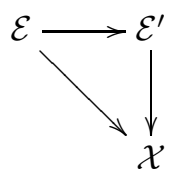

2-commutative. 
Conjecture 4.7. There exists a moduli space parameterizing isomorphism classes of twisted arcs of $\mathcal{X}$ : we will denote it by $\mathcal{J}_{\infty} \mathcal{X}$.

Let us suppose that this conjecture holds.

Lemma 4.8. Suppose that $\mathcal{X}$ is a quotient stack $[M / G]$ for $M$ and $G$ as above. Then we have

$$
\mathcal{J}_{\infty} \mathcal{X} \cong J_{\infty}^{G} M
$$

Proof. Let $E \rightarrow M$ be a $G$-arc. Taking the quotient stacks, we obtain a twisted arc

$$
[E / G] \rightarrow \mathcal{X}
$$

This defines a map $J_{\infty}^{G} M \rightarrow \mathcal{J}_{\infty} \mathcal{X}$. Conversely, for a twisted $\operatorname{arc} \mathcal{E} \rightarrow \mathcal{X}$, we put $E:=M \times_{\mathcal{X}} \mathcal{E}$. Then the map $E \rightarrow M$ is a $G$-arc.

Although not defining twisted jets, we expect:

Conjecture 4.9. For each $n \in \mathbb{Z}_{\geq 0}$, there exists a moduli space $\mathcal{J}_{n} \mathcal{X}$ of twisted $n$-jets of $\mathcal{X}$ satisfying:

(1) If $\mathcal{X}=[M / G]$, then $\mathcal{J}_{n} \mathcal{X}=J_{n}^{G} M$.

(2) There exist truncation maps $\mathcal{J}_{n+1} \mathcal{X} \rightarrow \mathcal{J}_{n} \mathcal{X}$ making $\mathcal{J}_{\infty} \mathcal{X}$ the projective limit of $\mathcal{J}_{n} \mathcal{X}, n \in \mathbb{Z}_{\geq 0}$.

Assuming these conjectures, when $\mathcal{X}$ is of pure dimension $d$ (relatively over $D$ ), we can define the motivic measure $\mu_{\mathcal{X}}$ on $\mathcal{J}_{\infty} \mathcal{X}$ in a similar way as the one on the arc space of a variety. The untwisting technique (Section 7) would provide an evidence that there are enough stable or measurable subsets of $\mathcal{J}_{\infty} \mathcal{X}$.

\section{Formulating the Change OF VARiables FORMUla FOR DM STACKS}

5.1. Maps of twisted arc spaces. Given a morphism $f: \mathcal{Y} \rightarrow \mathcal{X}$ of DM stacks of finite type over $D$, we can construct a map

$$
f_{\infty}: \mathcal{J}_{\infty} \mathcal{Y} \rightarrow \mathcal{J}_{\infty} \mathcal{X}
$$

as follows: let $\gamma:[E / G] \rightarrow \mathcal{Y}$ be a twisted arc with $E$ a connected $G$-cover of $D$. Let $y \in \mathcal{Y}$ be the image of the closed point by $\gamma$ and $x \in \mathcal{X}$ the image of $y$ by $f$. Set

$$
N:=\operatorname{Ker}(G \stackrel{\gamma}{\rightarrow} \operatorname{Aut}(y) \stackrel{f}{\rightarrow} \operatorname{Aut}(x)) .
$$

Then $f \circ \gamma$ factors as

$$
f \circ \gamma: \mathcal{E}=[E / G] \rightarrow \mathcal{E}^{\prime}=[(E / N) /(G / N)] \rightarrow \mathcal{X} .
$$

Here $[(E / N) /(G / N)]$ is the quotient stack associated to the induced action of the quotient group $G / N$ on the quotient scheme $E / N$. We define $f_{\infty}(\gamma)$ to be the induced morphism $\mathcal{E}^{\prime} \rightarrow \mathcal{X}$.

Lemma 5.1 (Almost bijectivity lemma). Let $f: \mathcal{Y} \rightarrow \mathcal{X}$ be a morphism of DM stacks of finite type over D. Suppose that for closed substacks $\mathcal{Y}^{\prime} \subset \mathcal{Y}$ and $\mathcal{X}^{\prime} \subset \mathcal{X}$, $f$ induces an isomorphism $\mathcal{Y} \backslash \mathcal{Y}^{\prime} \stackrel{\sim}{\rightarrow} \mathcal{X} \backslash \mathcal{X}^{\prime}$. Then $f_{\infty}$ restricts to a bijection

$$
\mathcal{J}_{\infty} \mathcal{Y} \backslash \mathcal{J}_{\infty} \mathcal{Y}^{\prime} \rightarrow \mathcal{J}_{\infty} \mathcal{X} \backslash \mathcal{J}_{\infty} \mathcal{X}^{\prime}
$$

Proof. Let $(\gamma: \mathcal{E} \rightarrow \mathcal{X}) \in \mathcal{J}_{\infty} \mathcal{X} \backslash \mathcal{J}_{\infty} \mathcal{X}^{\prime}$. Then we put $\tilde{\mathcal{E}}$ to be the normalization of the irreducible component of $\mathcal{E} \times \mathcal{X} \mathcal{Y}$ dominating $\mathcal{E}$. The induced morphism $\tilde{\gamma}: \tilde{\mathcal{E}} \rightarrow \mathcal{Y}$ is the unique twisted arc with $f_{\infty}(\tilde{\gamma})=\gamma$. This shows the lemma. 
If $\mathcal{Y}$ and $\mathcal{X}$ have pure dimension $d$, and if $\operatorname{dim} \mathcal{X}^{\prime}, \operatorname{dim} \mathcal{Y}^{\prime}<d$, then we expect that $\mathcal{J}_{\infty} \mathcal{X}^{\prime}$ and $\mathcal{J}_{\infty} \mathcal{Y}^{\prime}$ have measure zero as subsets of $\mathcal{J}_{\infty} \mathcal{X}$ and $\mathcal{J}_{\infty} \mathcal{Y}$ respectively. Then the lemma says that $f_{\infty}: \mathcal{J}_{\infty} \mathcal{Y} \rightarrow \mathcal{J}_{\infty} \mathcal{X}$ is almost bijective. Thanks to this, we can expect that motivic integrals on $\mathcal{J}_{\infty} \mathcal{X}$ are transformed into ones on $\mathcal{J}_{\infty} \mathcal{Y}$ and vice versa. The formula describing the transform will be called the change of variables formula.

5.2. Order functions associated to submodules. To formulate the change of variables formula, we need to introduce order functions on the space of twisted arcs.

Definition 5.2. Let $\mathcal{X}$ be a DM stack of pure dimension $d$, let $N \subset M$ be coherent $\mathcal{O}_{\mathcal{X}}$-modules which are generically of rank one. (In practice, we often take $\mathcal{O}_{\mathcal{X}}$, $\Omega_{\mathcal{X} / D}^{d}$ or $\omega_{\mathcal{X} / D}$ as $M$.) Then we define the order function of the submodule $N$

$$
\operatorname{ord} N: \mathcal{J}_{\infty} \mathcal{X} \rightarrow \mathbb{Q}_{\geq 0} \cup\{\infty\}
$$

as follows: let $\gamma:[E / G] \rightarrow \mathcal{X}$ be a twisted arc with $E$ a connected $G$-cover of $D$. If $N_{E}$ and $M_{E}$ denote the pull-backs of $N$ and $M$ to $E$, and if $M_{E} /$ tors is free of rank one, then

$$
\operatorname{Im}\left(N_{E} \rightarrow M_{E} / \text { tors }\right)=s^{n}\left(M_{E} / \text { tors }\right)
$$

for some $n \in \mathbb{Z}_{\geq 0}$ with $s$ a uniformizer of the function field of $E$. Then we define

$$
(\text { ord } N)(\gamma):=\frac{n}{\sharp G} \text {. }
$$

If $M_{E} /$ tors is not free of rank one, then we put $(\operatorname{ord} N)(\gamma)=\infty$, expecting that this does not happen for almost all $\gamma$.

Remark 5.3. Generalizing this, we can also define the order function of a fractional submodule $N$ of $M$, that is, an $\mathcal{O}_{\mathcal{X}}$-submodule of $M \otimes_{\mathcal{O}_{\mathcal{X}}} Q$ with $Q$ the sheaf of total quotient rings. Then the function may take negative values.

Definition 5.4. For a generically étale morphism $f: \mathcal{Y} \rightarrow \mathcal{X}$ of DM stacks, we define its Jacobian order function, ord $\mathrm{Jac}_{f}$, as the order function of the submodule $\operatorname{Im}\left(f^{*} \Omega_{\mathcal{X} / D}^{d} \rightarrow \Omega_{\mathcal{Y} / D}^{d}\right) \subset \Omega_{\mathcal{Y} / D}^{d}$.

5.3. The change of variables formula. The following is the main conjecture of this paper:

Conjecture 5.5 (Main Conjecture). For each DM stack $\mathcal{X}$ of finite type and pure dimension over $D$, there exists a canonically defined function

$$
w_{\mathcal{X}}: \mathcal{J}_{\infty} \mathcal{X} \rightarrow\left(\bigcup_{x \in \mathcal{X}} \frac{1}{\sharp \operatorname{Aut}(x)} \mathbb{Z}\right)
$$

such that if $\mathcal{X}$ is a scheme, then $w_{\mathcal{X}} \equiv 0$. (We call this the weight function on $\mathcal{J}_{\infty} \mathcal{X}$.) Moreover, for a proper birational morphism $f: \mathcal{Y} \rightarrow \mathcal{X}$ of such stacks, and for a measurable function $F: \mathcal{J}_{\infty} \mathcal{X} \supset C \rightarrow \frac{1}{r} \mathbb{Z} \cup\{\infty\}$, we have

$$
\int_{C} \mathbb{L}^{F+w_{\mathcal{X}}} d \mu_{\mathcal{X}}=\int_{f_{\infty}^{-1}(C)} \mathbb{L}^{F \circ f_{\infty}-\mathrm{ord} \mathrm{Jac}_{f}+w_{\mathcal{Y}}} d \mu_{\mathcal{Y}}
$$

This is basically of the same form as the formula in the tame setting proved in 31. However differences lie for instance in the facts that in the wild case, the moduli spaces of twisted arcs are much larger and that weight functions may take negative and unbounded values. The conjecture has been proved in 32 for the 
morphism from the quotient stack $\left[\mathbb{A}_{k}^{d} / G\right]$ to the quotient variety $\mathbb{A}_{k}^{d} / G$ associated to a linear action of $G=\mathbb{Z} / p \mathbb{Z}$ with $p=\operatorname{char}(k)$.

\section{More Details on the linear CASE}

Let $G \subset G L_{d}(A)$ be a finite subgroup, which acts on $V:=\mathbb{A}_{D}^{d}$ : we call $V$ a $G$-representation over $D$. Then we put $\mathcal{X}:=[V / G]$ and $X=V / G$, the quotient stack and variety. In this section, we will study the detailed structure of twisted arc and jet spaces and obtain a conjectural "explicit" expression of the weight function $w_{\mathcal{X}}$.

\subsection{Spaces of arcs and jets. Let}

$$
E=\operatorname{Spec} B \rightarrow D=\operatorname{Spec} A
$$

be a $G$-cover. We will study the structure of $J_{\infty}^{G, E} V$ and $\pi_{n}\left(J_{\infty}^{G, E} V\right), n \in \mathbb{Z}_{\geq 0}$, where $\pi_{n}$ are the truncation maps. Let $A\left[x_{1}, \ldots, x_{d}\right]$ be the coordinate ring of $V$. We have the induced $A$-linear action of $G$ on $A^{d}=\bigoplus_{i=1}^{d} A \cdot x_{i}$. This uniquely extends to a $B$-linear action on $B^{d}$. Let

$$
\rho: G \rightarrow \operatorname{Aut}_{B}\left(B^{d}\right)
$$

be the corresponding map. On the other hand, $G$ acts on $B$ and diagonally on $B^{d}$, which induces a map

$$
\delta: G \rightarrow \operatorname{Aut}_{A}\left(B^{d}\right) .
$$

Since $\rho(g), g \in G$ are represented by $d \times d$ matrices with entries from $A$, for every $g, g^{\prime} \in G, \rho(g)$ and $\delta\left(g^{\prime}\right)$ are commutative. Hence the map

$$
\rho \delta^{-1}: G \rightarrow \operatorname{Aut}_{A}\left(B^{d}\right), g \mapsto \rho(g) \circ \delta(g)^{-1}
$$

is a group homomorphism.

Definition 6.1. We define $\Xi_{B / A}^{V} \subset B^{d}$ to be the $G$-invariant subset for $\rho \delta^{-1}$, which is the locus where the two $G$-actions $\rho$ and $\delta$ coincide. For $n \in \mathbb{Z}_{\geq 0}$, writing $E_{n}=\operatorname{Spec} B_{n}$, we put $\Xi_{B / A, n}^{V}$ to be the image of $\Xi_{B / A}^{V}$ in $B_{n}^{d}$.

By definition, these modules $\Xi_{B / A}^{V}, \Xi_{B / A, n}^{V}$ have the unique natural $G$-action.

Lemma 6.2. We can identify $J_{\infty}^{G, E} V$ with $\Xi_{B / A}^{V} / G$ and $\pi_{n}\left(J_{\infty}^{G, E} V\right)$ with $\Xi_{B / A, n}^{V} / G$.

Proof. A $G$-arc $E \rightarrow V$ corresponds to an equivariant homomorphism

$$
A\left[x_{1}, \ldots, x_{d}\right] \rightarrow B .
$$

In turn, this corresponds to an element of $\Xi_{B / A}^{V}$. The set $J_{\infty}^{G, E} V$ is identified with the set of such equivariant homomorphisms modulo the $G$-action, in turn with $\Xi_{B / A}^{V} / G$. The assertion on jets follows from this.

Proposition 6.3. $\Xi_{B / A}^{V}$ is a free A-module of rank $d$. Moreover it is saturated, that is, if ax $\in \Xi_{B / A}^{V}$ with $a \in A$ and $x \in B^{d}$, then $x \in \Xi_{B / A}^{V}$.

Proof. For $a \in A$, the multiplication map, $B^{d} \rightarrow B^{d}, x \mapsto a x$, commutes with all $\rho(g)$ and $\sigma(g)$. This shows the saturatedness. The freeness follows from the facts that it is torsion-free and that $A$ is a PID. It remains to show that the rank is $d$.

The $G$-action $\rho \delta^{-1}$ makes $B^{d}$ a $G$-equivariant $B$-module. Let $L$ be the fraction field of $B$ and $p: \operatorname{Spec} L \rightarrow \operatorname{Spec} K$ the associated $G$-torsor. Then $\Xi_{B / A}^{V} \otimes_{A} K$ is 
identified with $\left(p_{*}\left(B^{d} \otimes_{B} L\right)\right)^{G}$. We can see that this has rank $d$, for instance, by trivializing the $G$-torsor with a base change.

From the proposition, we can identify $\Xi_{B / A, n}^{V}$ with affine spaces over $k$, using Witt vectors in the mixed characteristic case (for instance, see [6, page 276]). Now we can easily deduce the following:

Corollary 6.4. For every $n \in \mathbb{Z}_{\geq 0}, \pi_{n+1}\left(\mathcal{J}_{\infty} \mathcal{X}\right) \rightarrow \pi_{n}\left(\mathcal{J}_{\infty} \mathcal{X}\right)$ is an $\mathbb{L}^{d}$-fibration, where $\pi_{n}: \mathcal{J}_{\infty} \mathcal{X} \rightarrow \mathcal{J}_{n} \mathcal{X}$ is the truncation map.

\subsection{Weight functions in the linear case.}

Definition 6.5. Let $\alpha_{1}=\left(\alpha_{1 j}\right)_{1 \leq j \leq d}, \ldots, \alpha_{d}=\left(\alpha_{d j}\right)_{1 \leq j \leq d} \in B^{d}$ be an $A$-basis of $\Xi_{B / A}^{V}$ and put $Q:=\left(\alpha_{i j}\right) \in M_{d}(B)$. Let $G^{\prime} \subset G$ be the stabilizer of some connected component of $E$. Let $V_{0}:=V \times_{D} D_{0}$. Then we defins 3 a weight function $w=w_{V}: G-\operatorname{Cov}(D) \rightarrow \mathbb{Q}$ by

$$
\begin{aligned}
w(E): & =d-\frac{1}{\sharp G} \text { length } \frac{B}{(\operatorname{det} Q)}-\operatorname{dim} V_{0}^{G^{\prime}} \\
& =d-\frac{1}{\sharp G} \text { length } \frac{B^{d}}{B \cdot \Xi_{B / A}^{V}}-\operatorname{dim} V_{0}^{G^{\prime}} .
\end{aligned}
$$

Note that if $B$ is a domain, then the middle terms are written as $v_{B}(\operatorname{det} Q) / \sharp G$ with $v_{B}$ the normalized valuation of $B$.

Conjecture 6.6. For the quotient stack $\mathcal{X}=[V / G]$ associated to a $G$-representation $V$ over $D$, the weight function $w_{\mathcal{X}}$ from Conjecture 5.5 factors as

$$
w_{\mathcal{X}}: \mathcal{J}_{\infty} \mathcal{X}=J_{\infty}^{G} M \rightarrow G-\operatorname{Cov}(D) \stackrel{w_{V}}{\longrightarrow} \mathbb{Q} .
$$

We will show an evidence for the conjecture in the next section.

Example 6.7. Let $\zeta \in k$ be a primitive $m$-th root of 1 with $\operatorname{char}(k) \nmid m$ and $G:=\left\langle\operatorname{diag}\left(\zeta^{a_{1}}, \ldots, \zeta^{a_{d}}\right)\right\rangle \subset G L_{d}(k)$ with $0 \leq a_{i}<m$ and $\operatorname{gcd}\left(a_{1}, \ldots, a_{d}\right)$ prime to $m$. Then $G$ is cyclic of order $m$. Let

$$
E=\operatorname{Spec} k\left[\left[t^{1 / m}\right]\right] \rightarrow D=\operatorname{Spec} k[[t]]
$$

be a $G$-cover with $\sigma\left(t^{1 / m}\right)=\zeta t^{1 / m}$. Then for a natural basis of $\Xi$, the matrix in Definition 6.5 becomes

$$
Q=\operatorname{diag}\left(t^{a_{1} / m}, \ldots, t^{a_{d} / m}\right) .
$$

Hence

$$
\begin{aligned}
w(E) & =d-\frac{1}{m} \sum_{i=1}^{d} a_{i}-\sharp\left\{i \mid a_{i}=0\right\} \\
& =d-\frac{1}{m} \sum_{i=1}^{d} a_{i}^{\prime},
\end{aligned}
$$

where $a_{i}^{\prime}$ is an integer such that $a_{i}^{\prime} \equiv a_{i} \bmod m$ and $0<a_{i}^{\prime} \leq m$. This agrees with weight functions in [9, 30, 31].

\footnotetext{
${ }^{3}$ Afterwards it turned out that this definition is not quite correct; the term $\operatorname{dim} V_{0}^{G^{\prime}}$ needs to be modified, although they coincide in some cases; see [28].
} 
Example 6.8. Suppose that $\operatorname{char}(k)=p>0$ and $G=\langle\sigma\rangle$ is cyclic of order $p$. Also suppose that $G$ acts on $k\left[x_{1}, \ldots, x_{d}\right], d \leq p$ by

$$
\sigma\left(x_{i}\right)= \begin{cases}x_{i}+x_{i+1} & (i<d) \\ x_{d} & (i=d) .\end{cases}
$$

Let

$$
E=\operatorname{Spec} B \rightarrow D=\operatorname{Spec} k[[t]]
$$

be a $G$-cover with ramification jump $j>0$. Namely, if $s \in B$ is a uniformizer, then $v_{B}(\sigma(s)-s)=j+1$. (Note that $j$ is necessarily prime to $p$.) Then there exist $f_{0}, \ldots, f_{d-1} \in B$ such that

(1) $v_{B}\left(f_{i}\right) \equiv-i j \bmod p$ and $0 \leq v_{B}\left(f_{i}\right)<p$,

(2) If we put $\delta=\sigma^{*}-\operatorname{id}_{B}$, then

$$
v_{B}\left(\delta^{n}\left(f_{i}\right)\right)= \begin{cases}v_{B}\left(f_{i}\right)+n j & (0 \leq n \leq i) \\ \infty & (n>i)\end{cases}
$$

(3) $\left(\delta^{i}\left(f_{0}\right)\right)_{1 \leq i \leq d}, \ldots,\left(\delta^{i}\left(f_{d-1}\right)\right)_{1 \leq i \leq d} \in B^{d}$ form a basis of $\Xi_{B}$.

The matrix $Q=\left(\delta^{i}\left(f_{j}\right)\right)_{0 \leq i, j \leq d-1}$ corresponding to the basis is triangular and its determinant is $\prod_{i=0}^{d-1} \delta^{i}\left(f_{i}\right)$. Since

we have

$$
v_{B}\left(\delta^{i}\left(f_{i}\right)\right)=v_{B}\left(f_{i}\right)+i j=p \cdot\left\lceil\frac{i j}{p}\right\rceil
$$

$$
\begin{aligned}
w(E) & =d-\sum_{i=0}^{d-1}\left\lceil\frac{i j}{p}\right\rceil-1 \\
& =-\sum_{i=1}^{d-1}\left\lfloor\frac{i j}{p}\right\rfloor .
\end{aligned}
$$

This agrees with the weight function in 32 .

These examples would suggest that the tame part positively contributes to the weight and the purely wild part negatively does 4 and that the weight function measures how tame/wild a $G$-cover $E \rightarrow D$ is. The formula defining $w_{V}$ contains $\operatorname{det} Q$ and it is not clear how to compute it in general. Therefore we would like to ask:

Problem 6.9. Does $w(E)$ depend only on numerical invariants of $E$ and $V$ as in the above examples? If it is the case, find a formula 5

To attack the above problem, knowing properties of the weight function would be helpful. For instance, it is natural to expect the following properties:

(1) Let $E \rightarrow D$ be a $G$-cover, $E^{\prime} \subset E$ a connected component, $G^{\prime} \subset G$ its stabilizer and $V^{\prime}$ the same scheme as $V$ endowed with the induced $G^{\prime}$ action. Then $w_{V}(E)=w_{V^{\prime}}\left(E^{\prime}\right)$.

\footnotetext{
${ }^{4}$ Afterwards this phenomenon was also confirmed in the case of permutation representations in [27.

${ }^{5}$ Afterwards it turned out that $w(E)$ is described by discriminants for permutation representations [27] and for little more complicated ones [29. However the problem is still open for the general case.
} 
(2) For a trivial $G$-cover $E=\bigsqcup_{g \in G} D \rightarrow D, w_{V}(E)=0$.

(3) For $G$-representations $V$ and $W$ over $D$, let $G$ act on $V \times{ }_{D} W$ diagonally. Then $w_{V \times_{D} W}(E)=w_{V}(E)+w_{W}(E)$.

\section{UnTWisting: A JUSTIFICATION OF CONJECTURES}

In this section, we will try to justify Conjectures 5.5 and 6.6. Here we will use a technique which we call untwisting. This would reduce the change of variables formula for stacks to the one for varieties, which has been already mostly established. Such an argument was already used by Denef and Loeser [9]. Our argument will be somehow more involved than theirs. One of the reasons is that we have to untwist in the opposite direction. It is inevitable because the weight function may take negative values in the wild case 6

We will only consider the proper birational morphism

$$
\phi: \mathcal{X}=[M / G] \rightarrow X=M / G,
$$

associated to a $G$-variety $M$, as all keys seem to appear already in this situation. Then, like the change of variables formula in known cases, Conjecture 5.5 would basically follow from:

Conjecture 7.1 (Key lemma). Let $\gamma: \mathcal{E} \rightarrow \mathcal{X}$ be a twisted arc of $\mathcal{X}$ which sends the generic point of $\mathcal{E}$ into the isomorphism locus of $\phi$. Let $\phi_{n}: \pi_{n}\left(\mathcal{J}_{\infty} \mathcal{X}\right) \rightarrow J_{n} X$ be the natural map. Then for $n \gg 0$,

$$
\left[\phi_{n}^{-1}\left(\phi_{n}\left(\pi_{n}(\gamma)\right)\right)\right]=\mathbb{L}^{\operatorname{ord~Jac}_{\phi}(\gamma)-w_{\mathcal{X}}} .
$$

\subsection{Fixing a $G$-cover of $D$.}

Lemma 7.2. Let $\gamma, \gamma^{\prime} \in J_{\infty}^{G} M$ be $G$-arcs sending the generic points into the étale locus of the quotient map $M \rightarrow X$. If they have the same image in $J_{n} X$ for $n \gg 0$, then they have the same image in $G-\operatorname{Cov}(D)$.

Proof. Let $E \rightarrow D$ be the $G$-cover associated to $\gamma$. We consider only the case where $E$ is connected. We may suppose that $M=\operatorname{Spec} S$ and $X=\operatorname{Spec} R$ with $R=S^{G}$. Let $f \in R$ be such that $\gamma\left(E^{*}\right) \subset \operatorname{Spec} S_{f}$ and we can write

$$
S_{f}=R_{f}[x] /(h(x)) .
$$

Let $\bar{h}(x) \in K[x]$ to be the image of $h(x)$ by $R_{f}[x] \rightarrow K[x]$ derived from $\gamma$. Then the function field of $E$ is $L=K[x] /(\bar{h}(x))$. For $n \gg 0$, the induced $n$-jet $f_{n}\left(\pi_{n}(\gamma)\right)$ of $X$ determines the coefficients of $\bar{h}(x)$ up to sufficiently high order. Hence it determines $L$ and $E$.

7.2. The linear case. Now we consider the case where $M=V=\operatorname{Spec} A\left[x_{1}, \ldots, x_{d}\right]$ with a linear $G$-action. Fix $E=\operatorname{Spec} B \in G-\operatorname{Cov}(D)$. As in Definition 6.5, we take an $A$-basis of $\Xi_{B / A}^{V}$,

$$
\alpha_{1}=\left(\alpha_{1 j}\right)_{1 \leq j \leq d}, \ldots, \alpha_{d}=\left(\alpha_{d j}\right)_{1 \leq j \leq d} \in B^{d},
$$

\footnotetext{
${ }^{6}$ These two sentences were wrong. This misunderstanding caused the complicated presentation of the untwisting. However this was the way how the author reached the definition of weight functions and Conjecture 7.1 below. In [29 he revisited this technique in a more intrinsic way.
} 
which we now think of row vectors, and put

$$
Q:=\left(\begin{array}{c}
\alpha_{1} \\
\vdots \\
\alpha_{d}
\end{array}\right)=\left(\alpha_{i j}\right)_{1 \leq i, j \leq d} \in M_{d}(B),
$$

which is invertible over $B \otimes_{A} K$.

Definition 7.3. Let $t$ be a uniformizer of $A$ and let $l \in \mathbb{Z}_{>0}$ be such that $t^{l} Q^{-1} \in$ $M_{d}(B)$. Then $t^{l} Q^{-1}$ defines a $B$-linear transform on $B^{d} \cong \bigoplus B \cdot x_{j}$. Extending it, we obtain a $B$-algebra endomorphism $u^{*}$ of $B\left[x_{1}, \ldots, x_{d}\right]$. Putting $V_{E}:=$ Spec $B\left[x_{1}, \ldots, x_{d}\right]=V \times_{D} E$, we define a $B$-linear map

$$
u: V_{E} \rightarrow V_{E}
$$

to be the one corresponding to $u^{*}$. We call $u$ an untwisting map.

Suppose that $G$ acts on $V_{E}=V \times_{D} E$ diagonally. A $G$-arc $E \rightarrow V$ corresponds to a $G$-equivariant $E$-morphism $E \rightarrow V_{E}$. For $1 \leq i \leq d$, let $\gamma_{i}: E \rightarrow V_{E}$ be the $G$-equivariant $E$-morphism such that

$$
\begin{aligned}
&\left.\gamma_{i}^{*}\right|_{\oplus B \cdot x_{j}}: \bigoplus B \cdot x_{j} \rightarrow B \\
& \sum a_{j} x_{j} \mapsto \alpha_{i}\left(\begin{array}{c}
a_{1} \\
\vdots \\
a_{d}
\end{array}\right) .
\end{aligned}
$$

Then

$$
\begin{aligned}
&\left.\left(u \circ \gamma_{i}\right)^{*}\right|_{\oplus B \cdot x_{j}}: \bigoplus B \cdot x_{j} \rightarrow B \\
& \sum a_{j} x_{j} \mapsto \alpha_{i} t^{l} Q^{-1}\left(\begin{array}{c}
a_{1} \\
\vdots \\
a_{d}
\end{array}\right)=t^{l} a_{i} .
\end{aligned}
$$

For any $\gamma \in J_{\infty}^{G, E} V,\left.\gamma^{*}\right|_{\oplus B \cdot x_{j}}$ is an $A$-linear combination of $\left.\gamma_{i}^{*}\right|_{\oplus B \cdot x_{j}}$, and $(u \circ$ $\gamma)\left.^{*}\right|_{\oplus B \cdot x_{j}}$ is an $A$-linear combination of $\left.\left(u \circ \gamma_{i}\right)^{*}\right|_{\oplus B \cdot x_{j}}$. For $0 \leq n \leq \infty$, we denote the set of $G$-equivariant morphisms $E_{n} \rightarrow V$ by $\dot{J}_{n}^{G, E} V$, distinguishing it from $J_{n}^{G, E} V=\left(\dot{J}_{n}^{G, E} V\right) / G$. We have the identification $\dot{J}_{n}^{G, E} V=\Xi_{B / A, n}^{V}$ (see Lemma 6.2 and its proof).

Proposition 7.4. For $l \leq n \leq \infty$, let $J_{n}^{\geq l} V$ be the subset of $n$-jets $\gamma: D_{n} \rightarrow V$ of $V$ with $\gamma^{*}\left(x_{i}\right) \in \mathfrak{m}_{A_{n}}^{l}$, where $\mathfrak{m}_{A_{n}}$ is the maximal ideal of $A_{n}$. We have a natural bijection

$$
u_{\infty}: \dot{J}_{\infty}^{G, E} V \rightarrow J_{\infty}^{\geq l} V
$$

Moreover, for $l \leq n<\infty$, the induced map

$$
u_{n}: \pi_{n}\left(\dot{J}_{\infty}^{G, E} V\right) \rightarrow J_{n}^{\geq l} V
$$

is a trivial $\mathbb{A}_{k}^{c}$-fibration with $c=\operatorname{dim} V_{0}^{G^{\prime}}+d(l-1)$.

Proof. The first assertion is now obvious. The maps $u_{n}$ are linear surjections. The dimensions of their kernels are independent of $n$ and equal to 


$$
\begin{aligned}
c & =\operatorname{dim} J_{l}^{G, E} V-\operatorname{dim} J_{l}^{\geq l} V \\
& =\operatorname{dim} J_{0}^{G, E} V+d(l-1) \\
& =\operatorname{dim} V_{0}^{G^{\prime}}+d(l-1) .
\end{aligned}
$$

Here $G^{\prime} \subset G$ is the stabilizer of some connected component of $E$.

Since $u$ is an isomorphism outside the special fiber of $V_{E} \rightarrow D$, there exists a rational map $\psi \circ u^{-1}: V_{E} \rightarrow X_{E}$.

Lemma 7.5. The rational map $\psi \circ u^{-1}$ is defined over $D$.

Proof. Suppose that this is not true. Then there exists $f \in \operatorname{frac}\left(A\left[x_{1}, \ldots, x_{d}\right]^{G}\right)$ with $f \notin u^{*}\left(\operatorname{frac}\left(A\left[x_{1}, \ldots, x_{d}\right]\right)\right.$. Then, for general $\gamma \in J_{\infty}^{\leq l} V$, if $\gamma_{E}: E \rightarrow V_{E}$ is the morphism induced from $\gamma$ by extending scalars, then $\gamma_{E}^{*}(f) \notin K$. This contradicts the fact that $u_{\infty}^{-1}(\gamma): E \rightarrow V$ induces an $\operatorname{arc} D \rightarrow X$.

Let $\psi: V \rightarrow X$ be the quotient map and $\psi^{\prime}=\psi \circ u^{-1}: V \rightarrow X$ the above rational map over $D$.

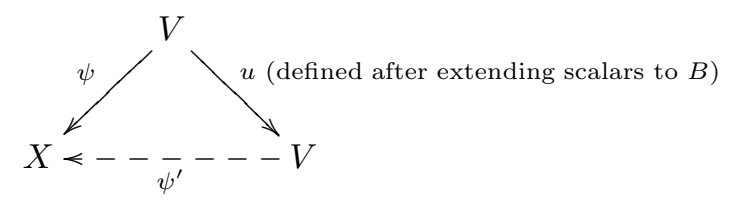

This diagram induces the commutative diagram of jet spaces:

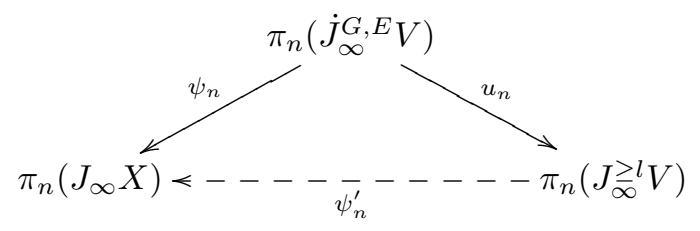

Here $\psi_{n}^{\prime}$ should be understood as the restriction of a "correspondence"

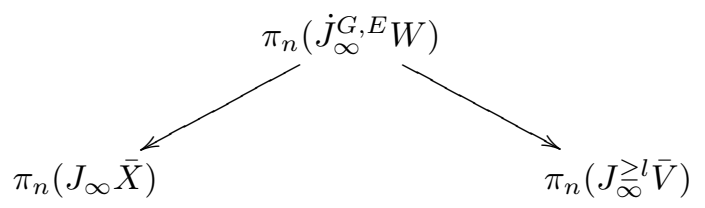

where $\bar{X}$ and $\bar{V}$ are compactifications of $X$ and $V$ respectively, and $W$ is a resolution of indeterminacies of $\psi^{\prime}: \bar{V} \rightarrow \bar{X}$.

We now fix a $G$-arc $\gamma \in \dot{J}_{\infty}^{G, E} E$ not contained in a bad locus. We denote the relative dimensions of morphisms $\psi_{n}$ and $u_{n}$ at jets derived from $\gamma$ by $\operatorname{dim} \psi_{n}$ and $\operatorname{dim} u_{n}$ respectively. Also we write the "relative dimension of $\psi_{n}^{\prime}$ " as $\operatorname{dim} \psi_{n}^{\prime}$. Then for $n \gg 0$,

$$
\operatorname{dim} \psi_{n}=\operatorname{dim} \psi_{n}^{\prime}+\operatorname{dim} u_{n}=\operatorname{dim} \psi_{n}^{\prime}+c
$$

with $c$ as above. On the other hand, we would have the associativity of Jacobian orders,

$$
\operatorname{ord} \mathrm{Jac}_{\psi^{\prime}}=\operatorname{ord} \mathrm{Jac}_{\psi}+\operatorname{ord} \mathrm{Jac}_{u^{-1}}
$$


where ord Jac? denotes the Jacobian order of ? at the arc derived from $\gamma$. Since $\mathrm{Jac}_{u^{-1}}=\operatorname{det}\left(t^{-l} Q\right)=t^{-d l} \cdot \operatorname{det} Q$ and $\operatorname{det} Q \in B$, we would have

$$
\text { ord } \mathrm{Jac}_{u^{-1}} \equiv \frac{1}{\sharp G} \text { length } \frac{B}{(\operatorname{det} Q)}-d l \text {. }
$$

On the other hand, suitably generalizing the change of variables formula to our rational map, we would have

$$
\operatorname{ord} \operatorname{Jac}_{\psi^{\prime}}=\operatorname{dim} \psi_{n}^{\prime}
$$

Hence

$$
\begin{aligned}
\operatorname{dim} \psi_{n} & =\operatorname{dim} \psi_{n}^{\prime}+c \\
& =\operatorname{ord} \operatorname{Jac}_{\psi}-w_{\mathcal{X}}(\gamma) .
\end{aligned}
$$

These arguments would justify Conjecture 7.1 and hence Conjectures 5.5 and 6.6 in this situation.

7.3. Untwisting: the non-linear or singular case. Next we consider the case where the $G$-variety $M$ is just an affine (possibly singular) $D$-variety 7 There exists an equivariant closed embedding $M \hookrightarrow V=\mathbb{A}_{D}^{d}$ with $G$ linearly acting on $V$. From $V$, we construct the same diagram as (7.2). Let $Y:=M / G$ and $\bar{M} \subset V$ the closure of $\left(\psi^{\prime}\right)^{-1}(Y)$. The bijection (7.1) restricts to a bijection

$$
\dot{J}_{\infty}^{G, E} M \rightarrow J_{\infty}^{\geq l} \bar{M}:=J_{\infty}^{\geq l} V \cap J_{\infty} \bar{M} .
$$

Diagram (7.3) restricts to:

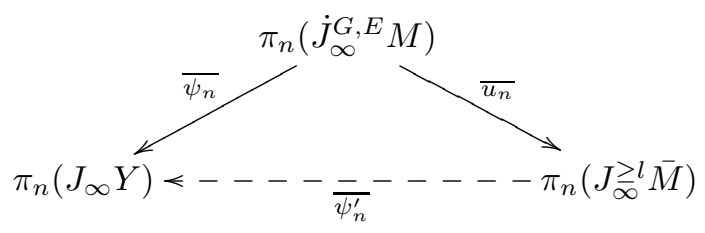

Here the overlines mean restrictions of maps. With similar notation as above, we would have

$$
\begin{aligned}
\operatorname{dim} \overline{\psi_{n}} & =\operatorname{dim} \overline{\psi_{n}^{\prime}}+\operatorname{dim} \overline{u_{n}} \\
& =\operatorname{ord} \operatorname{Jac}_{\bar{\psi}}+\operatorname{dim} \overline{u_{n}}+\operatorname{ord} \operatorname{Jac}_{\overline{u^{-1}}} .
\end{aligned}
$$

Therefore the weight of the relevant arc, $w_{[M / G]}(\gamma)$, would be

$$
-\operatorname{dim} \overline{u_{n}}-\operatorname{ord} \operatorname{Jac} \overline{u^{-1}} \text {. }
$$

Unlike the linear case, the two terms are not probably be constant even if we fix a $G$-cover $E$.

Remark 7.6. Another possible (and more direct) approach to the conjectures is to generalize Looijenga's argument [15]: we identify fibers of $\psi_{n}$ with suitable submodules of

$$
\operatorname{Hom}_{B}\left(\tilde{\gamma}^{*} \Omega_{\mathcal{Y} / \mathcal{X}}, \mathfrak{m}_{B}^{a} / \mathfrak{m}_{B}^{b}\right)
$$

and compute their dimensions.

\footnotetext{
${ }^{7}$ Afterwards this case was studied in [29] in more details.
} 


\section{Stringy motifs vs. Motivic MAsses of $G$-COVERS}

8.1. Stringy motifs. Let $\mathcal{X}$ be a normal $\mathbb{Q}$-Gorenstein DM stack over $D$. Namely for some $r>0$, the double dual $\omega_{\mathcal{X} / D}^{[r]}:=\left(\omega_{\mathcal{X} / D}^{\otimes r}\right)^{\vee \vee}$ of $\omega_{\mathcal{X} / D}^{\otimes r}$ is invertible. Then we define a function $F_{\mathcal{X}}$ on $\mathcal{J}_{\infty} \mathcal{X}$ as the order function of the submodule

$$
\left(\Omega_{\mathcal{X} / D}^{d}\right)^{\otimes r} / \text { tors } \subset \omega_{\mathcal{X} / D}^{[r]}
$$

divided by $r$.

Definition 8.1. Let $W \subset \mathcal{X} \times{ }_{D} D_{0}$ be a closed subset and $\left(\mathcal{J}_{\infty} \mathcal{X}\right)_{W}$ the preimage of $W$ by the natural map $\mathcal{J}_{\infty} \mathcal{X} \rightarrow \mathcal{X} \times{ }_{D} D_{0}$. We define the stringy motif of $\mathcal{X}$ along $W$ as the integral

$$
M_{\mathrm{st}}(\mathcal{X})_{W}:=\int_{\left(\mathcal{J}_{\infty} \mathcal{X}\right)_{W}} \mathbb{L}^{F_{\mathcal{X}}+w_{\mathcal{X}}} d \mu_{\mathcal{X}} \in \hat{\mathcal{M}} \cup\{\infty\} .
$$

We say that $\mathcal{X}$ is stringily log terminal along $W$ if $M_{\mathrm{st}}(\mathcal{X})_{W} \neq \infty$. If $W=\mathcal{X}$, we just write $M_{\text {st }}(\mathcal{X})$. For a stack $\mathcal{X}$ defined over $k$ and a closed subset $W \subset \mathcal{X}$, we define $M_{\text {st }}(\mathcal{X})_{W}$ as $M_{\text {st }}\left(\mathcal{X} \otimes_{k} k[[t]]\right)_{W}$

Since $F_{\mathcal{X}}$ comes from singularities of $\mathcal{X}$ and $\omega_{\mathcal{X}}$ from local actions of stabilizers, roughly $M_{\text {st }}(\mathcal{X})_{W}$ measures how $\mathcal{X}$ is far from a smooth variety along $W$.

Remark 8.2. We may generalize the above definition to log stacks, that is, pairs of a DM stack and a $\mathbb{Q}$-divisor on it. We will not enter this issue in this paper.

Remark 8.3. A $\mathbb{Q}$-Gorenstein variety $X$ is $\log$ terminal in the usual sense if it is stringily log terminal. The converse holds if the variety admits a log resolution. If $\mathcal{X}$ is tame and smooth, then $\mathcal{X}$ is stringily log terminal. In general, a smooth DM stack may not be stringily log terminal (see [32]).

We can compute stringy invariants using resolution data if a nice resolution exists.

Proposition 8.4. Let $f: Y \rightarrow X$ be a resolution of a log terminal variety $X$ over $k$ such that the relative canonical divisor $K_{f}$ is simple normal crossing, say written as $K_{f}=\sum_{i \in I} a_{i} E_{i}$. For a subset $J \subset I$, we set $E_{J}^{\circ}:=\bigcap_{i \in J} E_{i} \backslash \bigcup_{i \notin J} E_{i}$. Then

$$
M_{\mathrm{st}}(X)_{W}=\sum_{J \subset I}\left[E_{J}^{\circ} \cap f^{-1}(W)\right] \prod_{j \in J} \frac{\mathbb{L}-1}{\mathbb{L}^{a_{i}+1}-1} .
$$

In particular, if $f$ is crepant, that is, $K_{f}=0$, then $M_{\mathrm{st}}(X)_{W}=\left[f^{-1}(W)\right]$.

Remark 8.5. Originally stringy invariants were defined by Batyrev [2] using a formula as in Proposition 8.4. Denef and Loeser [9] found a way to express them as motivic integrals over the arc space of the given singular variety.

Remark 8.6. If Conjecture 5.5 holds, then we can show that the stringy motif is invariant under $K$-equivalences. Moreover we can generalize it to log stacks.

8.2. Motivic masses. We conjectured that $G-\operatorname{Cov}(D)$ has the tautological motivic measure $\tau$. To a $G$-representation $V$ over $D$, we have associated the weight function $w_{V}$ on $G-\operatorname{Cov}(D)$. 
Definition 8.7. The motivic mass of $G$-covers of $D$ with respect to $V$ is

$$
\operatorname{Mass}(G, D, V):=\int_{G-\operatorname{Cov}(D)} \mathbb{L}^{w_{V}} d \tau=\sum_{r \in \mathbb{Q}}\left[w_{V}^{-1}(r)\right] \mathbb{L}^{r} \in \hat{\mathcal{M}} \cup\{\infty\} .
$$

Remark 8.8. The motivic mass is analogous to weighted counts of extensions of a local field by Serre 23] and Bhargava [5. Serre proved a mass formula for totally ramified field extensions of a local field with finite residue field. Bhargava proved a similar formula by allowing étale extensions. Kedlaya's reinterpretation [13] of Bhargava's result in terms of Galois representation, and Wood's subsequent work 25] seem to be more directly related to our motivic invariant.

Proposition 8.9. We denote by 0 the origin of $V \times_{D} D_{0}$, and its images in $X:=$ $V / G$ and $\mathcal{X}:=[V / G]$ as well. Suppose that Conjectures $[5.5$ and $[6.6$ hold. Suppose also that $G$ has no reflection, equivalently, the quotient map $V \rightarrow X$ is étale in codimension one. Then

$$
M_{\mathrm{st}}(X)_{0}=M_{\mathrm{st}}(\mathcal{X})_{0}=\operatorname{Mass}(G, D, V) .
$$

Moreover, if there exists a crepant resolution $f: Y \rightarrow X$, then these invariants are also equal to $\left[f^{-1}(0)\right]$.

Proof. Let $\phi: \mathcal{X} \rightarrow X$ be the natural morphism. Then $F_{X} \circ \phi_{\infty}=\operatorname{ord~Jac}_{\phi}$. Hence our conjectures show

$$
\int_{\left(J_{\infty} X\right)_{0}} \mathbb{L}^{F_{X}} d \mu_{X}=\int_{\left(\mathcal{J}_{\infty} \mathcal{X}\right)_{0}} \mathbb{L}^{w_{\mathcal{X}}} d \mu_{\mathcal{X}}=\int_{G-\operatorname{Cov}(D)} \mathbb{L}^{w_{V}} d \tau
$$

and the proposition.

Remark 8.10. If $\operatorname{char}(k) \nmid \sharp G$, then $G$ - $\operatorname{Cov}(D)$ have exactly $\sharp \operatorname{Conj}(G)$ points and

$$
M_{\mathrm{st}}(X)_{0}=\operatorname{Mass}(G, D, V)=\sum_{[g] \in \operatorname{Conj}(G)} \mathbb{L}^{\text {age } g} .
$$

See (9.1). Hence, if $f: Y \rightarrow X$ is a crepant resolution and if $\chi_{c}(-)$ denotes the topological Euler characteristic (for compactly supported cohomology), then

$$
\chi_{c}\left(f^{-1}(0)\right)=\sharp \operatorname{Conj}(G) .
$$

Thus we recover a version of the McKay correspondence. The basically same result was conjectured by Reid and first proved by Batyrev [3] in arbitrary dimension (for the historical account, see [19]). Our approach is essentially due to Denef and Loeser [9] at least in characteristic zero.

Remark 8.11. Without assuming any conjecture, the proposition above has been established in [32] when $G=\mathbb{Z} / p \mathbb{Z}, A=k[[t]]$ and $V$ is defined over $k$. Also, a variant of the proposition, regarded as a point-counting realization, will be proved in a forthcoming paper $\left[27 \sqrt[8]{8}\right.$ when $G$ is the $n$-th symmetric group $S_{n}, V$ is the direct sum of two copies of the standard representation and $Y$ is the Hilbert scheme of $n$ points on the affine plane. In the same paper, it will turn out that this case is closely related to Bhargava's mass formula for étale extensions.

\footnotetext{
${ }^{8}$ This paper is now published online.
} 


\section{Equisingular FAMILIES AND UNIFORMity OF MOTIVIC MASSES}

Motivated by uniformity problems of Kedlaya [13] and Wood [25, we pose the following problem:

Problem 9.1. Given a family $V_{S} \rightarrow S$ of $G$-representations over a scheme $S$ with fibers $V_{s}, s \in S$, are $\operatorname{Mass}\left(G, D, V_{s}\right)$ independent of $s \in S$ ? What finite groups admit such a uniform family of representations?

Since $\hat{\mathcal{M}}$ depends on $k$, to make the problem precise, we have to take a suitable realization of motivic masses. However, in all known cases, they are rational functions in $\mathbb{L}$, and hence we do not have to worry about realization. We are mainly interested in the case where $S$ surjects onto $\operatorname{Spec} \mathbb{Z}$ and what happens around points of characteristic dividing $\sharp G$.

Proposition 8.9 links the problem to:

Problem 9.2. What families of quotient singularities are equisingular in the sense that they have the "same" stringy motif?

These problems are wide open. From now on, we will focus on $G$-representations with $G$ of prime order. In this case, there have been already explicit formulae for motivic masses and stringy motifs at least in equal characteristic. We will now recall them.

Let $G$ be a finite group of prime order $p$ and $V$ a $G$-representation of dimension $d$ over $k$. We first suppose that $p \neq \operatorname{char}(k)$. Fixing a primitive $p$-th $\operatorname{root} \zeta \in k$ of 1 , we write each element $g \in G$ as $\operatorname{diag}\left(\zeta^{a_{1}}, \ldots, \zeta^{a_{d}}\right), 0 \leq a_{i}<r$ for a suitable basis of $V$, and put

$$
\text { age } g:=\frac{1}{r} \sum_{i=1}^{d} a_{i}
$$

Then

$$
\operatorname{Mass}(G, D, V)=\sum_{g \in G} \mathbb{L}^{\text {age } g} .
$$

If $V$ has no reflection, then this is equal to $M_{\mathrm{st}}(X)_{0}$ for the corresponding quotient singularity $0 \in X$.

Next suppose that $p=\operatorname{char}(k)$. Then $V$ is the direct sum of indecomposable representations of dimensions $d_{1}, \ldots, d_{l}$ with $\sum_{i} d_{i}=d$. (Such a decomposition of $V$ is unique up to permutation, and we have $d_{i} \leq p$.) We put

$$
D_{V}:=\sum_{i=1}^{l} \frac{d_{i}\left(d_{i}-1\right)}{2}
$$

Then $\operatorname{Mass}(G, D, V) \neq \infty$ if and only if $D_{V} \geq p$. When one of the two equivalent conditions holds, then $V$ has no reflection and we have

$$
\operatorname{Mass}(G, D, V)=M_{\mathrm{st}}(X)_{0}=1+\frac{(\mathbb{L}-1)\left(\sum_{s=1}^{p-1} \mathbb{L}^{s+w(s)}\right)}{\mathbb{L}-\mathbb{L}^{p-D_{V}}}
$$

with

$$
w(s)=-\sum_{i=1}^{l} \sum_{j=1}^{d_{i}-1}\lfloor j s / p\rfloor
$$


The topological Euler characteristic realization of $\operatorname{Mass}(G, D, V)$ equals $p$ in the tame case and

$$
1+\frac{p-1}{D_{V}-p+1}
$$

in the wild case. This shows the following:

Proposition 9.3. If a family $V_{S} \rightarrow S \rightarrow$ Spec $\mathbb{Z}$ is uniform in the sense of Problem 9.1, then $D_{V_{p}}=p$ for every reduction $V_{p}$ of $V_{S}$ to characteristic $p$.

The following example is a partial converse.

Example 9.4. Let $G$ be a finite group of prime order $p$ and $V_{p}$ a $G$-representation in characteristic $p$. Then for a suitable basis, a generator $\sigma \in G$ is represented by a Jordan normal form $J$ with blocks

$$
J_{i}=\left(\begin{array}{ccccc}
1 & 1 & & & \\
& 1 & 1 & & \\
& & \ddots & \ddots & \\
& & & 1 & 1 \\
& & & & 1
\end{array}\right)
$$

of sizes $d_{i} \leq p$. Thus we may suppose that $V_{p}$ is defined over $\mathbb{F}_{p}$. Then we lift $J$ to the matrix $\tilde{J}$ over $R:=\mathbb{Z}[x] /\left(x^{p}-1\right)$ with blocks

$$
\tilde{J}_{i}=\left(\begin{array}{ccccc}
1 & 1 & & & \\
& x & 1 & & \\
& & \ddots & \ddots & \\
& & & x^{d_{i}-2} & 1 \\
& & & & x^{d_{i}-1}
\end{array}\right) .
$$

For every $i$, the reduction of $\tilde{J}_{i}$ to a characteristic $\neq p$ has $d_{i}$ distinct eigenvalues all of which are $p$-th roots of 1 . Hence $\tilde{J}_{i}^{p}=1$ and $\tilde{J}^{p}=1$. Therefore $\tilde{J}$ induces a $G$-representation $V_{R}$ lifting $V_{p}$ over $R$.

Now suppose that $D_{V_{p}}=\sum_{i} \frac{d_{i}\left(d_{i}-1\right)}{2}=p$. Then

$$
\operatorname{Mass}\left(G, D, V_{p}\right)=1+\sum_{s=1}^{p-1} \mathbb{L}^{s+w_{V_{p}}(s)}
$$

while for a reduction $V_{l}$ of $V_{R}$ to characteristic $l \neq p$,

$$
\operatorname{Mass}\left(G, D, V_{l}\right)=1+\sum_{s=1}^{p-1} \mathbb{L}^{\alpha(s)} .
$$


Here $\alpha(s)=\sum_{i=1}^{l} \sum_{j=1}^{d_{i}}\{j s / p\},\{-\}$ denoting the fractional part. We now claim that $\alpha(s)=s+w_{V_{p}}(s)$. Indeed,

$$
\begin{aligned}
s & =\frac{s \cdot D_{V_{p}}}{p} \\
& =\sum_{i=1}^{l} \sum_{j=1}^{d_{i}-1} \frac{s j}{p} \\
& =\sum_{i} \sum_{j}\left\lfloor\frac{s j}{p}\right\rfloor+\sum_{i} \sum_{j}\left\{\frac{s j}{p}\right\} \\
& =-w_{V_{p}}(s)+\alpha(s) .
\end{aligned}
$$

Hence, thinking of $\mathbb{L}$ as an indeterminate, the motivic mass $\operatorname{Mass}\left(G, D, V_{l}\right)$ is independent of the characteristic $l$.

Example 9.5. In the preceding example, suppose that $V_{p}=W^{\oplus p}$ with $W$ the unique indecomposable $G$-representation of dimension 2 (in characteristic $p$ ). Then for any characteristic $l$,

$$
\operatorname{Mass}\left(G, D, V_{l}\right)=\sum_{i=0}^{p-1} \mathbb{L}^{i}=\left[\mathbb{P}^{p-1}\right] .
$$

\section{Non-EXistence of Resolution of Singularities}

Stringy motifs of log terminal varieties have a lot of information on their resolutions of singularities if exist. A detailed knowledge of this invariant might lead to the non-existence of resolution of singularities in positive characteristic. Conjecture 5.5 enables us to reduce the stringy invariant of a quotient variety to that of the corresponding quotient stack, which would be easier to compute in some cases. For this purpose, we have to know what constraints the existence of resolution imposes on the invariant.

\subsection{Rationality and the Poincaré duality.}

Definition 10.1. For a $\mathbb{Q}$-Gorenstein $k$-variety $X$ and a closed subset $W \subset X$, we put

$$
P_{\mathrm{st}}(X, \Delta)_{W}:=P\left(M_{\mathrm{st}}(X, \Delta)_{W}\right) \in \bigcup_{r>0} \mathbb{Z}\left(\left(T^{-1 / r}\right)\right) .
$$

with $P$ the virtual Poincaré realization. If $W=X$, we omit the subscript $W$.

Definition 10.2. A rational function $f\left(T^{1 / r}\right) \in \mathbb{Z}\left(T^{1 / r}\right)$ in $T^{1 / r}$ is said to satisfy the d-dimensional Poincaré duality if

$$
T^{2 d} \cdot f\left(T^{-1 / r}\right)=f\left(T^{1 / r}\right) .
$$

Proposition 10.3. Let $X$ be $a \mathbb{Q}$-Gorenstein $k$-variety which is log terminal along $W$. Suppose that there exists a resolution $f: Y \rightarrow X$ such that $K_{f}$ is simple normal crossing. Then $P_{\mathrm{st}}(X)_{W}$ is a rational function. Moreover, if $X$ is proper and $W=X$, then $P_{\mathrm{st}}(X)$ satisfies the $d$-dimensional Poincaré duality with $d=\operatorname{dim} X$.

Proof. The rationality follows from Proposition 8.4. We can prove the Poincaré duality in the same way as Batyrev [2] did. 
10.2. Medusa singularities. Next, let us recall the following result due to Kerz and Saito:

Theorem 10.4 ([14]). Let $M$ be a smooth $k$-variety with a finite group $G$ acting on it,

$$
\pi: M \rightarrow X=M / G
$$

the quotient map. Let $S \subset X$ be a closed subset containing the singular locus such that the reduced preimage $T:=\pi^{-1}\left(X_{\text {sing }}\right)_{\text {red }}$ is smooth. Suppose that a functorial resolution of singularities holds over $k$. Let $f: Y \rightarrow X$ be a resolution such that $E=f^{-1}(S)$ (with reduced structure) is a simple normal crossing divisor and $f$ is an isomorphism over $X \backslash X_{\text {sing. }}$. (We will call such $f$ a log resolution of $X$ and $S$.) Then the dual complex $\Gamma(E)$ of $E$ is contractible.

Putting this conversely, if either

(1) there exists a $\log$ resolution $f: Y \rightarrow X$ of $X$ and $S$ with $\Gamma\left(f^{-1}(S)\right)$ not contractible, or

(2) $X$ and $S$ do not admit any $\log$ resolution $f: Y \rightarrow X$ of $X$ and $S$ with $\Gamma\left(f^{-1}(S)\right)$ contractible,

then a functorial resolution over $k$ never holds. Saito calls such singularities of $X$ Medusa singularities. Since stringy motifs have a lot of information on resolution, we might use them to find Medusa singularities (if exist) and disprove the functorial resolution. For this purpose, a key result is the following:

Proposition 10.5. Let $X$ be $a \mathbb{Q}$-Gorenstein variety which is stringily log terminal along a closed subset $S \subset X$. Let $f: Y \rightarrow X$ be a log resolution of $X$ and $S$ such that $K_{f}$ is a simple normal crossing divisor supported in $E:=f^{-1}(S)$. Suppose that there exists a closed subset $R \subset S$ such that $R$ is proper over $k$ and $F:=f^{-1}(R)$ is a normal crossing variety having the same dual complex as $E$. Then

$$
\chi(\Gamma(E))=\chi(\Gamma(F))=\left.P_{\mathrm{st}}(X)_{R}\right|_{T=0},
$$

where $\chi$ is the Euler characteristic.

Proof. The following is a modification of Batyrev's computation in [2]. Write $E=$ $\bigcup_{i \in I} E_{i}, F=\bigcup_{i \in I} E_{i}$ and $K_{Y / X}=\sum a_{i} E_{i}$. Since

$$
P\left(F_{J}^{\circ}\right)=\sum_{J \subset J^{\prime}}(-1)^{\sharp J-\sharp J^{\prime}} P\left(F_{J^{\prime}}\right),
$$

we have

$$
\begin{aligned}
P_{\mathrm{st}}(X)_{R} & =\sum_{\emptyset \neq J \subset I} P\left(F_{J}^{\circ}\right) \prod_{j \in J} \frac{T^{2}-1}{T^{2\left(a_{i}+1\right)}-1} \\
& =\sum_{\emptyset \neq J \subset I}\left(\sum_{J \subset J^{\prime}}(-1)^{\sharp J-\sharp J^{\prime}} P\left(F_{J^{\prime}}\right)\right) \prod_{j \in J} \frac{T^{2}-1}{T^{2\left(a_{i}+1\right)}-1} \\
& =\sum_{\emptyset \neq J \subset I} P\left(F_{J}\right)\left(\prod_{j \in J}\left(\frac{T^{2}-1}{T^{2\left(a_{i}+1\right)}-1}-1\right)-(-1)^{\sharp J}\right) .
\end{aligned}
$$

Substituting 0 for $T$, we obtain

$$
\sum_{\emptyset \neq J \subset I}(-1)^{\sharp J-1} \sharp \pi_{0}\left(F_{J}\right),
$$


where $\pi_{0}$ denotes the set of connected components. This clearly equals to the Euler characteristic of the dual complex of $F$ and to that of $E$.

Remark 10.6. Looking at finer realizations, we might able to capture finer invariants of $\Gamma(E)$.

Example 10.7. Let $G$ be the cyclic group of prime order $p>0$ and $V$ a $G$ representation over $k$ of characteristic $p>0$. Suppose that $V$ is isomorphic to the direct sum of $l$ indecomposable representations and that $V$ has no reflection. Then the invariant locus $V^{G} \subset V$ and the singular locus $X_{\text {sing }}$ of the quotient variety $X:=V / G$ are isomorphic to $\mathbb{A}_{k}^{l}$. The the additive group $\mathbb{G}_{a}^{l} \cong \mathbb{A}_{k}^{l}$ acts on $X$ and

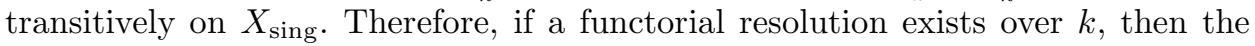
resolution $Y \rightarrow X$ of $X$ is $\mathbb{G}_{a}^{l}$-equivariant. Put $S=X_{\text {sing }}$ and $R$ to be any point of $S$. Suppose that $D_{V} \geq p$. Then the assumption of Proposition 10.5 holds, and from Equation 9.2

$$
\left.P_{\mathrm{st}}(X)_{R}\right|_{T=0}=1 \text {. }
$$

Hence we cannot see whether $\Gamma(E)$ is contractible or not in this case.

\subsection{Crepant resolutions.}

Problem 10.8. What quotient singularity admits a crepant resolution?

Crepant resolutions are certainly interesting, for instance, since they give a simple geometric meaning to stringy invariants and motivic masses. In characteristic zero, Ito [11, 12, Markushevich [16, 17, 4] and Roan [21, 20] proved that every threedimensional Gorenstein quotient singularity admits a crepant resolution. On the other hand, our knowledge is quite limited in the wild situation. As far as the author knows, the following are only known cases:

(1) Rational double points which are wild quotient singularities in Artin's classification [1. In this case, the minimal resolution is crepant.

(2) $G=S_{n}$ and $V$ is the direct sum of two copies of the standard representation. Then the Hilbert scheme of $n$ points on the affine plane is a crepant resolution of the quotient variety, which is isomorphic to the $n$-th symmetric product of the affine plane (see [7, page 229] for a historical account of this fact).

(3) $\operatorname{char}(k)=\sharp G=3$ and $V=V_{3}$, the 3-dimensional indecomposable representation. See [32].

We will be able to use stringy invariants also to study the above problem. For instance, we can show that the second example with $n=2$ and the last one are only possible cases for linear actions of a cyclic group of prime order in dimension $\leq 4$. It follows from the fact that $D_{V}=p$ is a necessary condition for the existence of crepant resolution. It is because otherwise the topological Euler characteristic realization is not an integer, which is not allowed if a crepant resolution exists. The same reasoning shows that if a crepant resolution exists, then $P_{\mathrm{st}}(X)$ is a polynomial in $T$ (not in $T^{1 / r}$ ).

\section{REFERENCES}

[1] M. Artin. Coverings of the rational double points in characteristic $p$. In Complex analysis and algebraic geometry, pages 11-22. Iwanami Shoten, Tokyo, 1977. 
[2] Victor V. Batyrev. Stringy Hodge numbers of varieties with Gorenstein canonical singularities. In Integrable systems and algebraic geometry (Kobe/Kyoto, 1997), pages 1-32. World Sci. Publ., River Edge, NJ, 1998.

[3] Victor V. Batyrev. Non-Archimedean integrals and stringy Euler numbers of log-terminal pairs. J. Eur. Math. Soc. (JEMS), 1(1):5-33, 1999.

[4] J. Bertin and D. Markushevich. Singularités quotients non abéliennes de dimension 3 et variétés de Calabi-Yau. Math. Ann., 299(1):105-116, 1994.

[5] Manjul Bhargava. Mass formulae for extensions of local fields, and conjectures on the density of number field discriminants. Int. Math. Res. Not. IMRN, (17):Art. ID rnm052, 20, 2007.

[6] Siegfried Bosch, Werner Lütkebohmert, and Michel Raynaud. Néron models, volume 21 of Ergebnisse der Mathematik und ihrer Grenzgebiete (3) [Results in Mathematics and Related Areas (3)]. Springer-Verlag, Berlin, 1990.

[7] Michel Brion and Shrawan Kumar. Frobenius splitting methods in geometry and representation theory, volume 231 of Progress in Mathematics. Birkhäuser Boston Inc., Boston, MA, 2005.

[8] Jan Denef and François Loeser. Germs of arcs on singular algebraic varieties and motivic integration. Invent. Math., 135(1):201-232, 1999.

[9] Jan Denef and François Loeser. Motivic integration, quotient singularities and the McKay correspondence. Compositio Math., 131(3):267-290, 2002.

[10] David Harbater. Moduli of p-covers of curves. Comm. Algebra, 8(12):1095-1122, 1980.

[11] Yukari Ito. Crepant resolution of trihedral singularities and the orbifold Euler characteristic. Internat. J. Math., 6(1):33-43, 1995.

[12] Yukari Ito. Gorenstein quotient singularities of monomial type in dimension three. J. Math. Sci. Univ. Tokyo, 2(2):419-440, 1995.

[13] Kiran S. Kedlaya. Mass formulas for local Galois representations. Int. Math. Res. Not. IMRN, (17):Art. ID rnm021, 26, 2007. With an appendix by Daniel Gulotta.

[14] Moritz Kerz and Shuji Saito. Cohomological Hasse principle and resolution of quotient singularities. New York J. Math., 19:597-645, 2013.

[15] Eduard Looijenga. Motivic measures. Astérisque, (276):267-297, 2002. Séminaire Bourbaki, Vol. 1999/2000.

[16] D. G. Markushevich, M. A. Olshanetsky, and A. M. Perelomov. Description of a class of superstring compactifications related to semisimple Lie algebras. Comm. Math. Phys., 111(2):247$274,1987$.

[17] Dimitri Markushevich. Resolution of $\mathbf{C}^{3} / H_{168}$. Math. Ann., 308(2):279-289, 1997.

[18] Johannes Nicaise and Julien Sebag. A note on motivic integration in mixed characteristic. arXiv:0912.4887, 2009.

[19] Miles Reid. La correspondance de McKay. Astérisque, (276):53-72, 2002. Séminaire Bourbaki, Vol. 1999/2000.

[20] Shi-Shyr Roan. On $c_{1}=0$ resolution of quotient singularity. Internat. J. Math., 5(4):523-536, 1994.

[21] Shi-Shyr Roan. Minimal resolutions of Gorenstein orbifolds in dimension three. Topology, 35(2):489-508, 1996.

[22] Julien Sebag. Intégration motivique sur les schémas formels. Bull. Soc. Math. France, 132(1):1-54, 2004.

[23] Jean-Pierre Serre. Une "formule de masse" pour les extensions totalement ramifiées de degré donné d'un corps local. C. R. Acad. Sci. Paris Sér. A-B, 286(22):A1031-A1036, 1978.

[24] Fabio Tonini. Stacks of ramified galois covers. Ph.D. thesis, arXiv:1307.1116.

[25] Melanie Matchett Wood. Mass formulas for local Galois representations to wreath products and cross products. Algebra Number Theory, 2(4):391-405, 2008.

[26] Melanie Matchett Wood and Takehiko Yasuda. Mass formulas for local Galois representations and quotient singularities II: dualities and resolution of singularities. arXiv:1505.07577.

[27] Melanie Matchett Wood and Takehiko Yasuda. Mass formulas for local Galois representations and quotient singularities I: a comparison of counting functions. International Mathematics Research Notices, doi: 10.1093/imrn/rnv074, 2015.

[28] Takehiko Yasuda. The wild McKay correspondence and $p$-adic measures. arXiv:1412.5260.

[29] Takehiko Yasuda. Wilder McKay correspondences. arXiv:1404.3373, to appear in Nagoya Mathematical Journal. 
[30] Takehiko Yasuda. Twisted jets, motivic measures and orbifold cohomology. Compos. Math., 140(2):396-422, 2004.

[31] Takehiko Yasuda. Motivic integration over Deligne-Mumford stacks. Adv. Math., 207(2):707761,2006

[32] Takehiko Yasuda. The p-cyclic McKay correspondence via motivic integration. Compos. Math., 150(7):1125-1168, 2014.

Department of Mathematics, Graduate School of Science, Osaka University, ToyONAKA, OSAKA 560-0043, JAPAN

E-mail address: takehikoyasuda@math.sci.osaka-u.ac.jp 